with infants. ${ }^{45}$ Our results showed a cerebrospinal fluid $\mathrm{pH}$ lower than the blood $\mathrm{pH}$ in healthy infants; higher $\mathrm{PCO}_{2}$ values in cerebrospinal fluid than in blood; and a lower concentration of bicarbonate in cerebrospinal fluid than in blood, which is in accordance with previous reports. ${ }^{4-6}$ Most infants with metabolic acidosis had normal or near normal $\mathrm{pH}$ in cerebrospinal fluid. It is reasonable to assume that the reason for this phenomenon is twofold-the lower value of $\mathrm{PCO}_{2}$ and the higher concentrations of bicarbonate in the cerebrospinal fluid of those infants suffering from metabolic acidosis, compared with infants in the control group. This shows that even in the presence of an acute metabolic acidosis with a low blood $\mathrm{pH}$ the cerebrospinal fluid often succeeds in maintaining a near normal $\mathrm{pH}$ value. ${ }^{78}$

The infants with an acute metabolic acidosis in their blood showed neurological signs and symptoms (coma, convulsions, or both) confirming the assumption that metabolic acidosis in blood can lead to a cerebrospinal fluid acid base disequilibrium, thus causing cerebral dysfunction. During the acute plasma metabolic acidosis the $\mathrm{pH}$ in cerebrospinal fluid may shift in the opposite direction from the extracellular $\mathrm{pH}$. This 'paradoxical' response seems to be related to the free permeability of the meningeal barrier to carbon dioxide gas, while hydrogen and bicarbonate equilibration across the membrane takes place slowly. Another hypothesis is that a child with any neurological sign such as convulsions may have an impaired ability to control $\mathrm{pH}$ in cerebrospinal fluid. We have not included such infants as our study was intended to emphasise the fact that acute metabolic acidosis caused by gastroenteritis can, by itselt, cause a disequilibrium in the cerebrospinal fluid $\mathrm{pH}$ with the emergence of a neurological disorder.

The results of the present work stress the importance of the careful correction of the metabolic acidosis in infants suffering from acute gastroenteritis and showing neurological signs. A rapid infusion of sodium bicarbonate may correct the blood $\mathrm{pH}$ but as a result the $\mathrm{PCO}_{2}$ in cerebrospinal fluid may rise, lowering the $\mathrm{pH}$ and endangering brain function.

\footnotetext{
References

I Siggard-Andersen O. Acid-base status of blood. J Clin Lab Invest 1963;70:1-34(Supplement).

${ }^{2}$ Posner JB, Plum F. Spinal fluid $\mathrm{pH}$ and neurological symptoms in systemic acidosis. N Engl J Med 1967;277:605-13.

${ }^{3}$ Beresford HR, Posner JB, Plum F. Changes in brain lactate during induced cerebral scizures. Arch Neurol 1969;20:243.

${ }^{4}$ Krauss AN, Thibcault DW, Auld PA. Acid-base balance in cerebro-spinal fluid of newborn infants. Biol Neonate 1972;21: 25-34.

5 Albert MS, Rahill WJ, Vega L, Winters RW. Acid-base changes in CSF of infants with metabolic acidosis. $N$ Engl J Med 1966;274:719-21.

6 Manfredi F. Acid-base relations between serum and CSF in man under normal and abnormal conditions. J Lab Clin Med 1962;59:128-36.

${ }^{7}$ Posner JB, Plum F. Protection of CSF pH and of the brain function during severe metabolic acidosis. Trans Am Neurol Assoc 1966;91:38-43.

${ }^{8}$ Siesjo BK, Kjallquist A. A new theory for the regulation of the extra-cellular $\mathrm{pH}$ in the brain. Scand J Clin Lab Invest 1969;24:1-9.
}

Correspondence to Dr Y Horovitz, Pediatric Department A, Central Emek Hospital, Afula 18101, Israel.

Received 4 December 1984

\title{
Raised intracranial pressure not detected by ultrasound
}

\section{W PILLING AND R E CUDMORE}

\section{Departments of Radiology and Surgery, Alder Hey Children's Hospital, Liverpool}

SUMmaRY Two patients with raised intracranial pressure responding to ventriculoperitoneal shunting are described in whom ultrasound failed to show dilated ventricles. The importance of not equating absence of ventricular dilatation with normal pressure and ventricular dilatation with high pressure is emphasised.

Ultrasound of the skull has become a routine examination in the neonate and infant with increasing head circumference or signs of raised intracra- nial pressure, or both. The technique has come to be relied upon for assessment of ventricular size, with timing of insertion of a shunt being strongly influenced by the size of the ventricles.

We report two patients without ventricular dilatation who had clinical signs of raised intracranial pressure and raised pressure on ventricular tap. In both relief of symptoms and signs followed ventriculoperitoneal shunting.

\section{Case reports}

Case 1. A thoracic meningocele was diagnosed at 
birth in this patient and the head circumference increased during the early weeks of life. Ultrasound examination of the head (Fig. 1) showed some ventricular asymmetry but no dilatation at 4 days, 6 weeks, and 10 weeks of age. Because of the continuing increase in the head circumference, a ventricular tap was performed at age 9 weeks; this showed a pressure of $250 \mathrm{~mm}$ of water at a depth of $2.5 \mathrm{~cm}$ from the skin surface. Pressure was measured under general anaesthetic with zeroing of the manometer to the skin surface at the site of insertion of the needle. A Hakim ventriculoperitoneal shunt was inserted without complications. After operation the previously bulging fontanelle became flat and the head circumference decreased slightly. At follow up at 5 months of age the head circumference had returned to within normal limits.

Case 2. This patient was born at 28 weeks' gestation and developed bilateral grade III intraventricular haemorrhages and subsequent progressive ventricular dilatation, confirmed by ultrasound at the referring hospital. The patient was transferred for insertion of a Holter ventriculoperitoneal shunt which was carried out without complications at 7 weeks of age.

At 18 weeks of age the patient developed a bulging anterior fontanelle and vomiting, suggestive of a block of the shunt system. The ventricular pressure measured by the standard technique under general anaesthesia was recorded at $170 \mathrm{~mm}$ of water, and the shunt system was revised with relief of the symptoms. Ultrasound before shunt revision showed no evidence of ventricular dilatation.

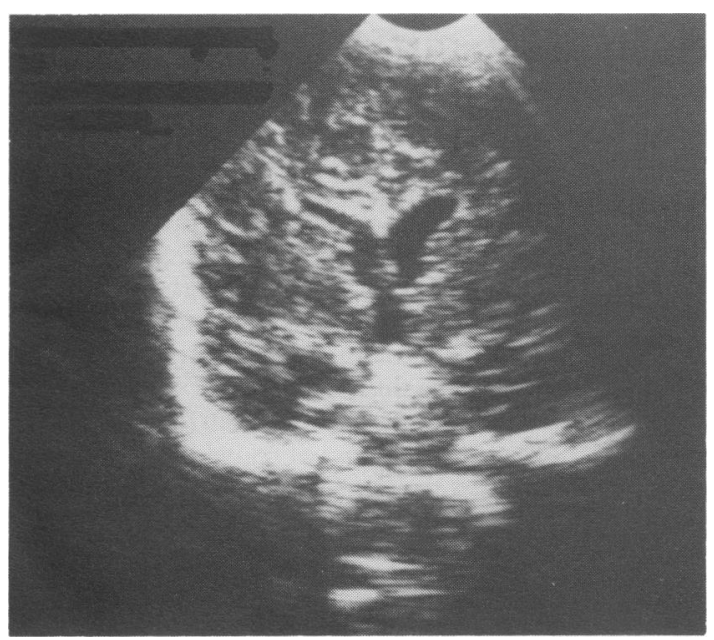

Fig. 1 Case 1: coronal section of ultrasound of brain showing ventricular asymmetry but no dilatation.

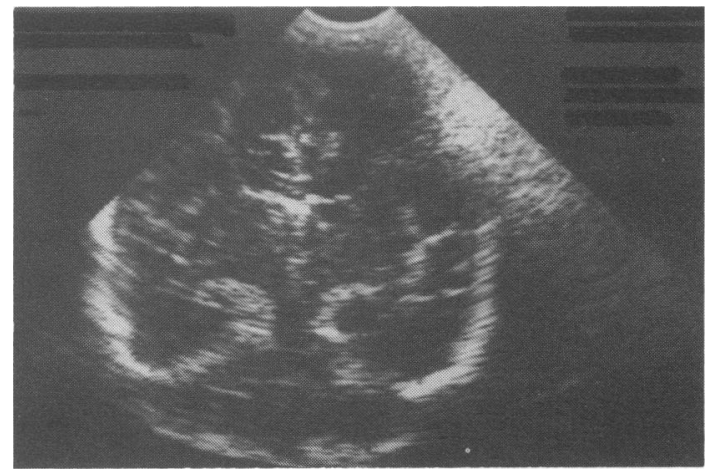

Fig. 2 Case 2: coronal section of ultrasound of brain showing normal lateral ventricles.

At the age of 5 months and again at 6 months the patient was readmitted with the same symptoms and attempted measurement of the pressure via needle insertion into the upper catheter of the shunt system showed no cerebrospinal fluid, diagnostic of blockage of the upper catheter of the shunt system. On neither occasion was ventricular dilatation detected by ultrasound, and symptoms were relieved by revision of the upper catheter.

At $7 \frac{1}{2}$ months of age the symptoms recurred and ultrasound examination on the same day (Fig. 2) showed no appreciable ventricular dilatation. At operation the following day the ventricles were difficult to find, they were presumed small and when entered with a needle the ventricular pressure was $200 \mathrm{~mm}$ of water. Revision of the shunt system led to resolution of the clinical signs and symptoms.

\section{Discussion}

It has become well recognised that ultrasound is an accurate method of assessing ventricular size, comparing very favourably with computed tomography. ${ }^{1}$ Many authors have accepted ventricular ratios of greater than $30 \%$ as indicative of hydrocephalus, ${ }^{2}$ equating ventricular dilatation with hydrocephalus, the former being purely descriptive of an appearance but the latter implying raised intracranial pressure.

It is important to realise that while large ventricles are most commonly associated with raised intracranial pressure, cerebral atrophy can cause similar ventricular dilatation, and likewise while normal size cerebral ventricles suggest normal intracranial pressure our two patients show that it is dangerous to assume this to be the case if clinical signs suggest otherwise. The reasons for this finding are uncertain. In both cases it seems most likely that the 
distribution of cerebrospinal fluid differs from that seen in most cases of hydrocephalus.

A possible explanation is that due to impairment of circulation of cerebrospinal fluid, in case 1 due to the Arnold-Chiari malformation and in case 2 due to adhesions after intraventricular haemorrhage, most of the expansion of cerebrospinal fluid spaces causing raised intracranial pressure occurs over the surface of the brain. This is quite difficult to detect by ultrasound but causes the same net effect to the brain as ventricular dilatation does in the more usual cases.

We think these two patients are unusual, but their cases illustrate the importance of not relying on the size of the ventricles to indicate raised intracranial pressure and the need for insertion or revision of a shunt system. Measurement of ventricular pressure is mandatory in any patient in whom there is raised intracranial pressure and whose ventricles are normal on ultrasound.

\section{References \\ ${ }^{1}$ Skolnick ML, Rosenbaum AE, Matzuli T, Guthkelch AN, Heinz ER. Detection of dilated cerebral ventricles in infants: a correlative study between ultrasound and CT. Radiology 1979;131:447-51. \\ 2 Johnson NL, Mack LA, Rumack CN, Frost M, Rashbaum C. B- mode echoencephalography in the normal and high risk infant. AJR 1979;153:375-81.}

Correspondence to Dr D W Pilling, Alder Hey Children's Hospital, Liverpool L12 2AP.

Received 14 February 1985

\title{
Hepatitis B virus replication in acute glomerulonephritis with chronic active hepatitis
}

\author{
P CADROBBI, F BORTOLOTTI, G ZACCHELLO, R RINALDI, M ARMIGLIATO, \\ AND G REALDI \\ Divisione di Malattie Infettive dell'Ospedale Civile, Clinica Medica $2 a$ e Clinica Pediatrica \\ dell'Università Padova, Italy.
}

SUMMARY A 3 year old boy who had chronic active hepatitis type $B$ with features of ongoing liver damage and active virus replication, developed acute membranous glomerulonephritis two years after the clinical onset of liver disease, when both hepatitis $\mathrm{B}$ e antigen and antibody were detectable in serum. After withdrawal of short term steroid treatment and resolution of hepatitis B virus replication, both glomerulonephritis and chronic hepatitis went into remission. Some months later hepatitis B surface antigen was no longer found in serum.

In recent years reports from different countries have emphasised the high prevalence of hepatitis B virus markers in the serum of children with membranous glomerulonephritis. ${ }^{12}$ On the other hand hepatitis B virus antigens together with immunoglobulins and complement components have been detected in the glomeruli of these patients, suggesting that hepatitis $B$ virus may be implicated in the pathogenesis of the disease. ${ }^{34}$ In most cases, however, hepatitis B virus infection remains asymptomatic until the development of membranous glomerulonephritis, although at this time a variety of associated liver disorders has been reported. ${ }^{23}$ Therefore no clear cut relation has emerged between the natural history of the infection and of the associated liver disease and the development of membranous glomerulonephritis. We described the clinical and virological features and the unusual course of chronic active hepatitis type B in a child who developed membranous glomerulonephritis two years after the clinical onset of liver disease.

\section{Case report}

This boy, the first child of a 27 year old mother, was born after an uncomplicated pregnancy and delivery. He was well until the age of 18 months (July 1979) when he presented at Padova Hospital with abdominal pain that subsided spontaneously. On that occasion routine tests showed increased transaminase concentrations (Figure), and hepatitis B surface and $e$ antigen positivity by radioimmunoassay. Both urine analysis and blood urea nitrogen were normal. No risk factors for hepatitis B virus infection emerged from the clinical history of the patient and both parents were hepatitis B surface antigen negative. After discharge the patient remained asymptomatic. He was next seen in March 1980 when his mother developed acute hepatitis type B. At this time the child was an asymptomatic, well developed, and well nourished child with mild hepatosplenomegaly. Transaminase activities were 